\title{
HYERS-ULAM STABILITY OF A NONLINEAR MULTI- TERM FRACTIONAL DIFFERENTIAL EQUATION ON BOUNDED TIME INTERVAL
}

\section{HUICHOL CHOI, YONGDO RI, PAK SUN AE and KINAM SIN}

Faculty of Mathematics

Kim II Sung University

Pyongyang

Democratic People's Republic of Korea

e-mail: 15bf12025@hit.edu.cn

\begin{abstract}
In this paper, we study the Hyers-Ulam stability of a nonlinear multi-term fractional differential equation with Caputo derivative on bounded time interval. First we discuss the existence and uniqueness for the solutions of initial value problem of a nonlinear multi-term fractional differential equation and second we give a criteria for Hyers-Ulam stability which is our main purpose. The technique relies on the fixed point theorem in the Banach space and the equivalence between Chebyshev norm and Bielecki norm.
\end{abstract}

\section{Introduction}

Nowadays, it became widely known that fractional differential equations serve a strong tool in modelling many phenomena in various fields such as engineering, economics, chemistry, etc. For more details

2010 Mathematics Subject Classification: 34A08, 34G20.

Keywords and phrases: Hyers-Ulam stability, fractional differential equation, Chebyshev norm, Bielecki norm.

Received January 22, 2019

(C) 2019 Scientific Advances Publishers 
on fractional differential equations, one can see the monographs of Kilbas et al. [1], Podlubny [2], and Miller and Ross [3]. The research of the fractional differential equations is more difficult than that of integer-order differential equations, because they are nonlocal and have weakly singular kernels.

Recently, one of the most active research topics of fractional differential equations is the Ulam stability theory. Ulam stability of differential equations is very important, since it guarantees the existence of solution and provides the theoretical basis of approximate solution. Obloza [4] initiated the study of the Ulam stability of differential equations and after that several results of the Ulam stability of classical differential equations were given by many researchers [5-12].

In recent years, the study of the Ulam stability of fractional differential equations is proceeding widely and many remarkable results on that have come out. Wang et al. [13] discussed the Hyers-Ulam stability and the Hyers-Ulam-Rassias stability of the fractional differential equation

$$
{ }^{c} D^{\alpha} x(t)=f(t, x(t)), \quad t \in[a, b],
$$

where $\alpha \in(0,1)$ and ${ }^{c} D^{\alpha}$ is Caputo fractional derivative of order $\alpha$. Wang et al. [14] obtained the criteria for the Hyers-Ulam stability and Hyers-Ulam-Rassias stability of fractional differential equation

$$
{ }^{c} D_{a+}^{\alpha} y(x)=F(x, y(x)), \quad t \in I,
$$

where $I$ is a given interval and $\alpha \in(0,1)$.

Wang and $\mathrm{Xu}$ [15] proved the Hyers-Ulam stability of two types of fractional linear differential equations

$$
\begin{gathered}
\left({ }^{c} D_{0+}^{\alpha} y\right)(x)-\lambda y(x)=f(x), \\
\left({ }^{c} D_{0+}^{\alpha} y\right)(x)-\lambda\left({ }^{c} D_{0+}^{\beta} y\right)(x)=g(x),
\end{gathered}
$$


where $x>0, \lambda \in(-\infty,+\infty), 0<\beta<\alpha, f(x)$ and $g(x)$ are real functions defined on $\mathbf{R}_{+}$. Mohamed [16] studied the Ulam stability of fractional impulsive differential equations by using singular Gronwall inequality. Besides, in many papers, the Ulam stability of classical differential equations has been extended to the several types of fractional differential equations. For more details, one can see [17-22].

Although there are many articles and papers on the Ulam stability of fractional differential equations, to our best knowledge, most results on that are restricted to monomial fractional differential equations. So we discuss the Hyers-Ulam stability of the following nonlinear multiterm fractional differential equation on bounded time interval.

$$
{ }^{c} D_{0+}^{\alpha} u(t)=f\left(t, u(t),{ }^{c} D_{0+}^{\beta} u(t)\right)
$$

where ${ }^{c} D_{0+}^{\alpha}$ is the Caputo fractional derivative of order $\alpha \in(1,2), 0<\beta<\alpha$ and $0<t<b<+\infty$.

The rest of this paper is consisted as follows: In Section 2, we introduce the basic definitions and lemmas which are used in this paper. In Section 3, we give the criteria for the Hyers-Ulam stability of the Equation (1).

\section{Preliminaries}

Definition 2.1. The fractional integral of order $\alpha>0$ with the lower limit $a$ for function $f \in L_{1}[a, b]$ is defined as

$$
I_{a+}^{\alpha} f(t):=\frac{1}{\Gamma(\alpha)} \int_{a}^{t}(t-\tau)^{\alpha-1} f(\tau) d \tau, \quad a \leq t \leq b,
$$

where $\Gamma($.$) is the Gamma function.$ 
Definition 2.2. The Caputo fractional derivative of order $\alpha>0$ for function $f$ is defined as

$$
{ }^{c} D_{a+}^{\alpha} f(t):=I_{a+}^{n-\alpha}\left[D^{n} f(t)\right]=\frac{1}{\Gamma(n-\alpha)} \int_{a}^{t} \frac{f^{(n)}(\tau)}{(t-\tau)^{\alpha-n+1}} d \tau,
$$

where $f^{(n)} \in L_{1}[a, b], n=[\alpha]+1$ and $[\alpha]$ is integer part of $\alpha$.

Lemma 2.1. For a function $f(x)=(x-a)^{p}$, let $p>-1, \alpha>0$. Then

$$
I_{a+}^{\alpha} f(t)=\frac{\Gamma(p+1)}{\Gamma(\alpha+p+1)}(t-a)^{\alpha+p} .
$$

Lemma 2.2. If $\alpha \in \boldsymbol{R}_{+}, n-1<\alpha \leq n, n \in N, f \in A C^{n}[a, b]$, then

$$
I_{a+}^{\alpha}{ }^{c} D_{a+}^{\alpha} f(t)=f(t)-\sum_{j=0}^{n-1} \frac{f^{(j)}(a)}{j !}(t-a)^{j}
$$

Definition 2.3. A function $u(t)$ such that ${ }^{c} D_{0+}^{\alpha} u \in C[0, b]$ is called solution of Equation (1) if it satisfies Equation (1).

Definition 2.4. Equation (1) is Hyers-Ulam stable if for a function $u(t)$ satisfying the following inequality:

$$
\left\|^{c} D_{0+}^{\alpha} u(t)-f\left(t, u(t),{ }^{c} D_{0+}^{\beta} u(t)\right)\right\|_{C[0, b]} \leq \varepsilon,
$$

and for some $\varepsilon>0$, there exist a solution $u_{0}(t)$ of the Equation (1) such that

$$
\left\|u-u_{0}\right\|_{C[0, b]} \leq K(\varepsilon),
$$

where $K(\varepsilon)$ is only dependent on $\varepsilon$ and $\|\cdot\|_{C[0, b]}$ is the Chebyshev norm.

Definition 2.5. $u(t)$ such that ${ }^{c} D_{0+}^{\alpha} u \in C[0, b]$ is called solution of inequality (2) if it satisfies Equation (2). 


\section{Hyers-Ulam Stability of Nonlinear Multi-term Fractional Differential Equation}

Let $u(t)$ be the solution of inequality (2). If we define $h(t)$ as below,

$$
h(t):={ }^{c} D_{0+}^{\alpha} u(t)-f\left(t, u(t),{ }^{c} D_{0+}^{\beta} u(t)\right), t \in[0, b],
$$

then $\|h\|_{C[0, b]} \leq \varepsilon$.

Lemma 3.1. Let us suppose $u(t)$ is a solution of inequality (2). Then $\nu(t)$ such that ${ }^{c} D_{0+}^{\alpha} u(t)=\nu(t)$ satisfies the following fractional integral equation:

$\nu(t)-f\left(t, u(0)+u^{\prime}(0) t+I_{0+}^{\alpha} \nu(t),{ }^{c} D_{0+}^{\beta}\left(u^{\prime}(0) t\right)+I_{0+}^{\alpha-\beta} \nu(t)\right)=h(t), t \in[0, b]$.

Conversely, $u(t)$ such that

$$
u(t)=u(0)+u^{\prime}(0) t+I_{0+}^{\alpha} \nu(t)
$$

is a solution of inequality (2), where $\nu(t)$ satisfies Equation (5) in $C[0, b]$.

Proof. First, we prove the first part of the lemma.

From the hypothesis of the first part, $u(t)$ is a solution of inequality (2) and

$$
{ }^{c} D_{0+}^{\alpha} u(t)=\nu(t) .
$$

${ }^{c} D_{0+}^{\alpha} u(t) \in C[0, b]$, so we can apply fractional integral operator $I_{0+}^{\alpha}$ to the both sides of the equality (7). Then we have the following equalities:

$$
\begin{gathered}
I_{0+}^{\alpha}{ }^{c} D_{0+}^{\alpha} u(t)=I_{0+}^{\alpha} \nu(t), \\
u(t)-u(0)-u^{\prime}(0) t=I_{0+}^{\alpha} \nu(t),
\end{gathered}
$$




$$
\begin{gathered}
u(t)=u(0)+u^{\prime}(0) t+I_{0+}^{\alpha} \nu(t), \\
{ }^{c} D_{0+}^{\beta} u(t)={ }^{c} D_{0+}^{\beta}\left(u^{\prime}(0) t\right)+I_{0+}^{\alpha-\beta} \nu(t) .
\end{gathered}
$$

By substituting above equalities into (4), Equation (5) is obtained.

Now, we prove the second part of the lemma.

$\nu(t)$ is a solution of Equation (5) and $u(t)=u(0)+u^{\prime}(0) t+I_{0+}^{\alpha} \nu(t)$. By applying the fractional differential operators ${ }^{c} D_{0+}^{\beta},{ }^{c} D_{0+}^{\alpha}$ to the both sides of (6), the following equalities are obtained:

$$
\begin{gathered}
{ }^{c} D_{0+}^{\beta} u(t)={ }^{c} D_{0+}^{\beta}(u(0)+u(0) t)+{ }^{c} D_{0+}^{\beta} I_{0+}^{\alpha} \nu(t)={ }^{c} D_{0+}^{\beta}\left(u^{\prime}(0) t\right)+I_{0+}^{\alpha-\beta} \nu(t), \\
{ }^{c} D_{0+}^{\alpha} u(t)={ }^{c} D_{0+}^{\alpha}(u(0)+u(0) t)+{ }^{c} D_{0+}^{\alpha} I_{0+}^{\alpha} \nu(t)=\nu(t) .
\end{gathered}
$$

From the Equation (5) and equalities (7)-(9), we have equality (4).

Lemma 3.2. Under the assumption:

$\left(\mathrm{H}_{0}\right)$ There exist $l_{1}>0, l_{2}>0$ such that $\left|f\left(t, x_{1}, y_{1}\right)-f\left(t, x_{2}, y_{2}\right)\right|$ $\leq l_{1}\left|x_{1}-x_{2}\right|+l_{2}\left|y_{1}-y_{2}\right|$ for any $\left(x_{1}, y_{1}\right),\left(x_{2}, y_{2}\right) \in R^{2}$.

Then the following nonlinear fractional integral equation has the unique solution in $C[0, b]$.

$$
\nu(t)-f\left(t, u(0)+u^{\prime}(0) t+I_{0+}^{\alpha} \nu(t),{ }^{c} D_{0+}^{\beta}\left(u^{\prime}(0) t\right)+I_{0+}^{\alpha-\beta} \nu(t)\right)=0, t \in[0, b] .
$$

Proof. Let us consider the following iterative scheme:

$$
\nu_{n+1}(t)=f\left(t, u(0)+u^{\prime}(0) t+I_{0+}^{\alpha} \nu_{n}(t), g(t)+I_{0+}^{\alpha-\beta} \nu_{n}(t)\right),
$$

where $g(t)={ }^{c} D_{0+}^{\beta}\left(u^{\prime}(0) t\right)$. 
Indeed, $\left\{v_{n}(t)\right\}$ is Cauchy sequence in $C[0, b]$. By considering the assumption $\left(\mathrm{H}_{0}\right)$,

$$
\begin{aligned}
& \left|v_{n+1}(t)-v_{n}(t)\right| \\
& =\mid f\left(t, u(0)+u^{\prime}(0) t+I_{0+}^{\alpha} \nu_{n}(t), g(t)+I_{0+}^{\alpha-\beta} \nu_{n}(t)\right)-f\left(t, u(0)+u^{\prime}(0) t\right. \\
& \left.+I_{0+}^{\alpha} \nu_{n-1}(t), g(t)+I_{0+}^{\alpha-\beta} \nu_{n-1}(t)\right) \\
& \leq l_{1}\left|I_{0+}^{\alpha} \nu_{n}(t)-I_{0+}^{\alpha} \nu_{n-1}(t)\right|+l_{2}\left|I_{0+}^{\alpha-\beta} \nu_{n}(t)-I_{0+}^{\alpha-\beta} \nu_{n-1}(t)\right| .
\end{aligned}
$$

Now we use the following Bielecki norm which is equivalent to Chebyshev norm.

$$
\|x\|_{k}:=\max \left\{e^{-k t}|x(t)|\right\}, x \in C[0, b], k>0 .
$$

Then the following inequalities hold:

$$
\begin{aligned}
\left|I_{0+}^{\alpha} \nu_{n}(t)-I_{0+}^{\alpha} \nu_{n-1}(t)\right| & \leq I_{0+}^{\alpha}\left|\nu_{n}(t)-v_{n-1}(t)\right|=I_{0+}^{\alpha} e^{-k t}\left|\nu_{n}(t)-v_{n-1}(t)\right| e^{k t} \\
& \leq\left\|v_{n}-v_{n-1}\right\|_{k} I_{0+}^{\alpha} e^{k t} \leq\left\|\nu_{n}-v_{n-1}\right\|_{k} \frac{e^{k t}}{k^{\alpha}} .
\end{aligned}
$$

In the same way, we obtain

$$
\left|I_{0+}^{\alpha-\beta} v_{n}(t)-I_{0+}^{\alpha-\beta} v_{n-1}(t)\right| \leq\left\|v_{n}-v_{n-1}\right\|_{k} \frac{e^{k t}}{k^{\alpha-\beta}} .
$$

Therefore, we have

$$
\left|v_{n+1}(t)-v_{n}(t)\right| \leq\left\|v_{n}-v_{n-1}\right\|_{k} e^{k t}\left(\frac{l_{1}}{k^{\alpha}}+\frac{l_{2}}{k^{\alpha-\beta}}\right),
$$

and by multiplying $e^{-k t}$ to the both sides,

$$
\left\|v_{n+1}-v_{n}\right\|_{k} \leq\left\|v_{n}-v_{n-1}\right\|_{k}\left(\frac{l_{1}}{k^{\alpha}}+\frac{l_{2}}{k^{\alpha-\beta}}\right)
$$

is obtained. 
Now we choose $k_{*}:=k$ such that $p=:\left(\frac{l_{1}}{k^{\alpha}}+\frac{l_{2}}{k^{\alpha-\beta}}\right)<1$, then the following relation holds:

$$
\left\|v_{n+1}-v_{n}\right\|_{k_{*}} \leq p\left\|v_{n}-v_{n-1}\right\|_{k_{*}} .
$$

So $\left\{v_{n}(t)\right\}$ converges as $n \rightarrow \infty$ and the limit $\nu_{*}(t)$ is the unique solution of Equation (10).

Lemma 3.3. If $w(t)$ is the unique solution of Equation (10), then the function

$$
u_{0}(t):=u(0)+u^{\prime}(0) t+I_{0+}^{\alpha} w(t)
$$

is a solution of Equation (1), where $w(t)$ is the unique solution of Equation (10).

Proof. From the assumption of the lemma, the following equality holds:

$$
w(t)-f\left(t, u(0)+u^{\prime}(0) t+I_{0+}^{\alpha} w(t),{ }^{c} D_{0+}^{\beta}\left(u^{\prime}(0) t\right)+I_{0+}^{\alpha-\beta} w(t)\right)=0, t \in[0, b] .
$$

By applying the fractional differential operator ${ }^{c} D_{0+}^{\alpha},{ }^{c} D_{0+}^{\beta}$ to the both sides of (11):

$$
\begin{gathered}
{ }^{c} D_{0+}^{\beta} u_{0}(t)={ }^{c} D_{0+}^{\beta}\left(u^{\prime}(0) t\right)+I_{0+}^{\alpha-\beta} w(t), \\
{ }^{c} D_{0+}^{\alpha} u_{0}(t)=w(t) .
\end{gathered}
$$

By substituting (11), (13), and (14) into (12), the following equality is obtained:

$$
{ }^{c} D_{0+}^{\alpha} u_{0}(t)=f\left(t, u_{0}(t),{ }^{c} D_{0+}^{\beta} u_{0}(t)\right), t \in[0, b] .
$$


Theorem 3.4. Under the assumption $\left(\mathrm{H}_{0}\right)$, the Equation (1) is Hyers-Ulam stable.

Proof. For any $\varepsilon>0$, let $u(t)$ be the solution of inequality (2). From the definition of fractional differential inequality, ${ }^{c} D_{0+}^{\alpha} u(t)$ exists and so $u(0)$ and $u^{\prime}(0)$ also exist. Therefore, from the Lemma 3.1, $u(t)$ is denoted as below.

$$
u(t)=u(0)+u^{\prime}(0) t+I_{0+}^{\alpha} \nu(t)
$$

where $\nu(t)$ is the solution of Equation (5) and

$$
h(t):={ }^{c} D_{0+}^{\alpha} u(t)-f\left(t, u(t),{ }^{c} D_{0+}^{\beta} u(t)\right), t \in[0, b] .
$$

On one side, from Lemma 3.2, fractional integral equation

$$
w(t)-f\left(t, u(0)+u^{\prime}(0) t+I_{0+}^{\alpha} w(t),{ }^{c} D_{0+}^{\beta}\left(u^{\prime}(0) t\right)+I_{0+}^{\alpha-\beta} w(t)\right)=0, t \in[0, b]
$$

has the unique solution. So from Lemma 3.3, a function $u_{0}(t)$ such that

$$
u_{0}(t):=u(0)+u^{\prime}(0) t+I_{0+}^{\alpha} w(t)
$$

is the solution of Equation (1).

Now, we estimate $\left\|u-u_{0}\right\|_{C[0, b]}$. From (6) and (16), we have

$$
\left|u(t)-u_{0}(t)\right|=\left|I_{0+}^{\alpha} \nu(t)-I_{0+}^{\alpha} w(t)\right| .
$$

By using Bielecki norm as in the proof of Lemma 3.2,

$$
\left|I_{a+}^{\alpha} \nu(t)-I_{a+}^{\alpha} w(t)\right| \leq I_{a+}^{\alpha}|\nu(t)-w(t)|=I_{a+}^{\alpha} e^{k t} e^{-k t}|\nu(t)-w(t)| \leq\|\nu(t)-w(t)\|_{k} I_{a+}^{\alpha} e^{k t} .
$$

From the inequality, the following inequality holds:

$$
\left|I_{0+}^{\alpha} \nu(t)-I_{0+}^{\alpha} w(t)\right| \leq\|\nu-w\|_{k} \frac{e^{k t}}{k^{\alpha}} .
$$


By multiplying $e^{-k t}$ to the both sides of (18), we obtain

$$
\left\|I_{0+}^{\alpha} \nu-I_{0+}^{\alpha} w\right\|_{k} \leq \frac{1}{k^{\alpha}}\|\nu-w\|_{k} .
$$

From (17) and (19),

$$
\left\|u-u_{0}\right\|_{k}=\left\|I_{0+}^{\alpha} \nu-I_{0+}^{\alpha} w\right\|_{k} \leq \frac{1}{k^{\alpha}}\|\nu-w\|_{k} .
$$

From (5) and (15), $|\nu(t)-w(t)|$ is estimated as the following:

$$
\begin{aligned}
|\nu(t)-w(t)| \leq & \mid f\left(t, u(0)+u^{\prime}(0) t+I_{0+}^{\alpha} \nu(t),{ }^{c} D_{0+}^{\beta}\left(u^{\prime}(0) t\right)+I_{0+}^{\alpha-\beta} \nu(t)\right) \\
& -f\left(t, u(0)+u^{\prime}(0) t+I_{0+}^{\alpha} w(t),{ }^{c} D_{0+}^{\beta}\left(u^{\prime}(0) t\right)+I_{0+}^{\alpha-\beta} w(t)\right)|+| h(t) \mid \\
\leq & l_{1}\left|I_{0+}^{\alpha} \nu(t)-I_{0+}^{\alpha} w(t)\right|+l_{2}\left|I_{0+}^{\alpha-\beta} \nu(t)-I_{0+}^{\alpha-\beta} w(t)\right|+|h(t)| \\
\leq & l_{1}\|\nu-w\|_{k} \frac{e^{k t}}{k^{\alpha}}+l_{2}\|\nu-w\|_{k} \frac{e^{k t}}{k^{\alpha-\beta}}+|h(t)|, t \in[0, b] .
\end{aligned}
$$

Therefore,

$$
\begin{gathered}
e^{-k t}|\nu(t)-w(t)| \leq l_{1}\|\nu-w\|_{k} \frac{1}{k^{\alpha}}+l_{2}\|\nu-w\|_{k} \frac{1}{k^{\alpha-\beta}}+e^{-k t}|h(t)|, \\
\|\nu-w\|_{k} \leq\left(\frac{l_{1}}{k^{\alpha}}+\frac{l_{2}}{k^{\alpha-\beta}}\right)\|\nu-w\|_{k}+\|h\|_{k} \leq \frac{l_{1}+l_{2}}{k^{\alpha-\beta}}\|\nu-w\|_{k}+\|h\|_{k} .
\end{gathered}
$$

Now we can easily investigate there exists $k_{*}>0$ such that $q:=\frac{l_{1}+l_{2}}{{k_{*}}^{\alpha-\beta}}>0$,

$$
\begin{aligned}
1-q>0, \frac{1}{k_{*}{ }^{\alpha}} \frac{1}{1-q} \leq 1 . & \text { Then } \\
& \|\nu-w\|_{k_{*}} \leq \frac{1}{1-q}\|h\|_{k_{*}},
\end{aligned}
$$


and from (20) and (21), the following inequalities hold:

$$
\left\|u-u_{0}\right\|_{k_{*}} \leq\left(\frac{1}{k_{*}^{\alpha}} \frac{1}{1-q}\right)\|h\|_{k_{*}} \leq\|h\|_{k_{*}} .
$$

The following inequalities show us the equivalence of Chebyshev norm and Bielecki norm.

$$
e^{-k b}\|x\|_{C[0, b]} \leq\|x\|_{k} \leq\|x\|_{C[0, b]} .
$$

Then

$$
e^{-k_{*} b}\left\|u-u_{0}\right\|_{C[0, b]} \leq\left\|u-u_{0}\right\|_{k_{*}} \leq\|h\|_{k_{*}} \leq\|h\|_{C[0, b]},
$$

and therefore,

$$
\left\|u-u_{0}\right\|_{C[0, b]} \leq e^{k_{*} b}\|h\|_{C[0, b]}
$$

From $\|h\|_{C[0, b]} \leq \varepsilon$, we obtain the following result:

$$
\left\|u-u_{0}\right\|_{C[0, b]} \leq K \varepsilon, K=e^{k_{*} b} .
$$

(13) shows us the Equation (1) is Hyers-Ulam stable.

Theorem 3.5. Let $D_{1}:=\left\{k \mid k>\left(l_{1}+l_{2}\right)^{1 /(\alpha-\beta)}\right\}$ and $D_{2}:=\{k \mid b<\ln$ $\left.\left(k^{\alpha}-k^{\beta}\left(l_{1}+l_{2}\right)\right)^{1 / k}\right\}$. If $D_{1} \cap D_{2} \neq \phi$, then (23) is estimated as the following:

$$
\left\|u-u_{0}\right\|_{C[0, b]} \leq \varepsilon
$$

Proof. $D_{1} \cap D_{2} \neq \phi$, so if we select $k_{*}$ in $D_{1} \cap D_{2}$, then $1-q>0$ from the definition of $D_{1}$, where

$$
q=\frac{l_{1}+l_{2}}{k_{*}^{\alpha-\beta}} .
$$


On one side, the following relations hold:

$$
\begin{aligned}
b & <\ln \left({k_{*}}^{\alpha}-k_{*}^{\beta}\left(l_{1}+l_{2}\right)\right)^{1 / k_{*}} \\
& \Leftrightarrow k_{*} b<\ln \left({k_{*}}^{\alpha}-{k_{*}}^{\beta}\left(l_{1}+l_{2}\right)\right) \\
& \Leftrightarrow 0<-k_{*} b+\ln \left(k_{*}^{\alpha}-k_{*}^{\beta}\left(l_{1}+l_{2}\right)\right) \\
& \Leftrightarrow 1<e^{-k_{*} b}\left({k_{*}}^{\alpha}-k_{*}^{\beta}\left(l_{1}+l_{2}\right)\right) \\
& \Leftrightarrow 1<e^{-k_{*} b} k_{*}^{\alpha}(1-q) \\
& \Leftrightarrow \frac{1}{{k_{*}}^{\alpha}} \frac{1}{1-q}<e^{-k_{*} b} .
\end{aligned}
$$

From (20) and (21),

$$
\left\|u-u_{0}\right\|_{k_{*}} \leq\left(\frac{1}{k_{*}^{\alpha}} \frac{1}{1-q}\right)\|h\|_{k_{*}} \leq e^{-k_{*} b}\|h\|_{k_{*}},
$$

and due to the equivalence of Chebyshev norm and Bielecki norm,

$$
e^{-k_{*} b}\left\|u-u_{0}\right\|_{C[0, b]} \leq\left\|u-u_{0}\right\|_{k_{*}} \leq e^{-k_{*} b}\|h\|_{k_{*}} \leq e^{-k_{*} b}\|h\|_{C[0, b]} .
$$

Therefore, we have

$$
\left\|u-u_{0}\right\|_{C[0, b]} \leq e^{-k_{*} b} e^{k_{*} b}\|h\|_{C[0, b]}=\|h\|_{C[0, b]},
$$

and by considering $\|h\|_{C[0, b]} \leq \varepsilon$, inequality (24) holds.

Remark 3.6. In (23), $K$ is a kind of characteristic of Hyers-Ulam stability. Theorem 3.5 shows us $K=1$ in some strict condition.

\section{Conclusion}

In this paper, we give a criteria for the Hyers-Ulam stability of a nonlinear multi-term fractional differential equation on bounded interval by using fixed point theorem in Banach space, Chebyshev norm 
and Bielecki norm. We think this method can be extended to the various differential equations. In the similar way, we can also expect the affirmative results on the Hyers-Ulam stability of fractional impulsive differential equations and fractional differential equations with $p$-Laplacian.

\section{References}

[1] A. A. Kilbas, H. M. Srivastava and J. J. Trujillo, Theory and Applications of Fractional Differential Equations, Elsevier, Amsterdam, 2006.

[2] K. S. Miller and B. Ross, An Introduction to the Fractional Calculus and Fractional Differential Equations, John, Wiley, 1993.

[3] I. Podlubny, Fractional Differential Equations, Academic Press, San Diego, 1999.

[4] M. Obloza, Hyers stability of the linear differential equation, Rocznik Nauk.Dydakt. Prace Mat. 13 (1993), 259-270.

[5] C. Alsina and R. Ger, On some inequalities and stability results related to the exponential function, Journal of Inequalities and Applications 2(4) (1998), 373-380.

[6] T. Miura, On the Hyers-Ulam stability of a differentiable map, Scientiae Mathematicae Japonicae 55(1) (2002), 17-24.

[7] T. Miura, S. E. Takahasi and H. Choda, On the Hyers-Ulam stability of real continuous function valued differentiable map, Tokyo Journal of Mathematics 24(2) (2001), 467-476.

DOI: https://doi.org/10.3836/tjm/1255958187

[8] S. E. Takahasi, H. Takagi, T. Miura and S. Miyajima, The Hyers-Ulam stability constants of first order linear differential operators, Journal of Mathematical Analysis and Applications 296(2) (2004), 403-409.

DOI: https://doi.org/10.1016/j.jmaa.2003.12.044

[9] M. Obloza, Connections between Hyers and Lyapunov stability of the ordinary differential equations, Rocznik Nauk.-Dydakt. Prace Mat. 14 (1997), 141-146.

[10] S. M. Jung, Hyers-Ulam stability of linear differential equations of first order, Applied Mathematics Letters 17(10) (2004), 1135-1140.

DOI: https://doi.org/10.1016/j.aml.2003.11.004

[11] S. M. Jung, Hyers-Ulam stability of linear differential equations of first order, III, Journal of Mathematical Analysis and Applications 311(1) (2005), 139-146.

DOI: https://doi.org/10.1016/j.jmaa.2005.02.025 
[12] S. M. Jung, Hyers-Ulam stability of linear differential equations of first order, II, Applied Mathematics Letters 19(9) (2006), 854-858.

DOI: https://doi.org/10.1016/j.aml.2005.11.004

[13] J. Wang, L. Lv and Y. Zhou, Ulam stability and data dependence for fractional differential equations with Caputo derivative, Electronic Journal of Qualitative Theory of Differential Equations 63 (2011), 1-10.

DOI: https://doi.org/10.14232/ejqtde.2011.1.63

[14] J. Wang, L. Lv and Y. Zhou, New concepts and results in stability of fractional differential equations, Communications in Nonlinear Science and Numerical Simulation 17(6) (2012), 2530-2538.

DOI: https://doi.org/10.1016/j.cnsns.2011.09.030

[15] C. Wang and T. Z. Xu, Hyers-Ulam stability of fractional linear differential equations involving Caputo fractional derivatives, Applications of Mathematics 60(4) (2015), 383-393.

DOI: https://doi.org/10.1007/s10492-015-0102-x

[16] Mohamed I. Abbas, Ulam stability of fractional impulsive differential equations with Riemann-Liouville integral boundary conditions, Journal of Contemporary Mathematical Analysis 50(5) (2015), 209-219.

DOI: https://doi.org/10.3103/S1068362315050015

[17] J. Wang, Y. Zhou and Michal Feckan, Nonlinear impulsive problems for fractional differential equations and Ulam stability, Computers \& Mathematics with Applications 64(10) (2012), 3389-3405.

DOI: https://doi.org/10.1016/j.camwa.2012.02.021

[18] Z. P. Yang, T. Z. Xu and M. Qi, Ulam-Hyers stability for fractional differential equations in quaternionic analysis, Advances in Applied Clifford Algebras 26(1) (2016), 469-478.

DOI: https://doi.org/10.1007/s00006-015-0576-3

[19] N. Eghbali, V. Kalvandi and J. M. Rassias, A fixed point approach to the MittagLeffler-Hyers-Ulam stability of a fractional integral equation, Open Mathematics 14(1) (2016), 237-246.

DOI: https://doi.org/10.1515/math-2016-0019

[20] R. W. Ibrahim and H. A. Jalab, Existence of Ulam stability for iterative fractional differential equations based on fractional entropy, Entropy 17(5) (2015), 3172-3181.

DOI: https://doi.org/10.3390/e17053172 
[21] Y. H. Shen and W. Chen, Laplace transform method for the Ulam stability of linear fractional differential equations with constant coefficients, Mediterranean Journal of Mathematics 14(1) (2017), 1-17.

DOI: https://doi.org/10.1007/s00009-016-0835-0

[22] J. F. Jiang, D. Q. Cao and H. T. Chen, The fixed point approach to the stability of fractional differential equations with causal operators, Qualitative Theory of Dynamical Systems 15(1) (2016), 3-18.

DOI: https://doi.org/10.1007/s12346-015-0136-1 